\title{
Megakaryocytic morphology in Janus kinase 2 V617F positive myeloproliferative neoplasm
}

\author{
Shuchi Ghai, Sharada Rai
}

\begin{abstract}
Context: Alterations in megakaryocyte morphology are the hallmark of myeloproliferative neoplasms (MPNs). These neoplasm are also associated with Janus kinase 2 (JAK2) V6I7F mutation in nearly $95 \%$ patients with polycythemia vera (PV), $40 \%$ patients of essential thrombocythemia (ET) and $50 \%$ patients of myelofibrosis (MF). The utility of megakaryocyte morphology in these disorders in correlation with JAK2 V6I7F remains unresolved. Aims: The aim of the study was to assess the morphology of megakaryocytes in bone marrow aspirates (BMAs) and bone marrow biopsies of patients of BCR-ABL negative MPNs with JAK2 V6I7F mutation. Settings and Design: This study was a retrospective and prospective, hospital-based study undertaken for a period ranging from January 20I I to April 2015. Subjects and Methods: Assessment of morphological features of megakaryocytes in I5 BMAs and their respective biopsies which included seven cases of PV, three cases of ET, and five cases of MF with JAK2 V6I7F mutation. Statistical Analysis Used: Chi-square test and Fisher exact test were used to compare the different features of megakaryocytes. Software version SPSS I3.0 was used. Results: Megakaryocytes in ET were found to have characteristically large size with staghorn multinucleated nuclei and exhibiting large amount of cytoplasm. MF showed dense clustering of megakaryocytes with staghorn nucleus along with sinusoidal dilatation and intrasinusoidal hematopoiesis. PV showed loose and dense clustering of megakaryocytes with a predominance of cloud-like nuclei. Few of the megakaryocytic morphologic features showed overlap between MF and PV and between ET and early MF. Conclusions: Megakaryocytic morphology can aid in the accurate diagnosis of the different subcategories of MPNs. This would help in categorization of clinically suspicious patients of JAK2 V6I7F negative patients.
\end{abstract}

Key words: Janus kinase 2 V6I7F, megakaryocyte, myeloproliferative neoplasm

\section{Introduction}

Myeloproliferative neoplasm (MPN) comprises of clonal stem cell disorders of multipotent hematopoietic progenitors. In 2008, the World Health Organization (WHO) revised the classification system for MPN. This classification reflected a paradigm shift from previous schemes as genetic information was incorporated with morphologic, cytochemical, immunophenotypic, and clinical information into diagnostic algorithms for myeloid neoplasms. ${ }^{[1]}$ The newer classification system incorporates mutations discovered in the Janus kinase 2 (JAK2) V617F and MPL genes. ${ }^{[2]}$

In 2005, three myeloproliferative diseases polycythemia vera (PV), essential thrombocythemia (ET), and myelofibrosis (MF) were associated with somatic mutation in JAK2. This finding led to a better understanding of the pathogenesis of these diseases. JAK2 V617F tyrosine kinase encoded by gain of function mutation in the gene JAK2 V617F present on short arm of chromosome 9 is known to be present in $95 \%$ patients with PV, $50 \%$ patients with MF and $40 \%$ patients with ET. ${ }^{[3]}$ The discovery of JAK2 V617F and similar mutations has revolutionized the diagnostic approach to the BCR-ABL negative MPNs, particularly PV, ET, and MF. This and the JAK exon 12 mutation and the mutations of MPL are important in differentiating between a neoplastic and reactive marrow proliferation. ${ }^{[4]}$

The role of megakaryocytic morphology has been emphasized in literature, but the terminology for megakaryocytic description is riddled with confusion and overlaps leading to under utility of megakaryocytic features and reliance on genetic markers. In this study, we used specific terminology for megakaryocytic description, attempting to identify specific megakaryocytic features for a particular entity. ${ }^{[5]}$

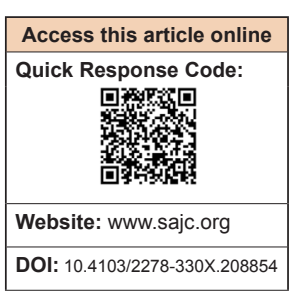

Department of Pathology, Kasturba Medical College,

Mangalore, Karnataka, India

Correspondence to: Dr. Sharada Rai,

E-mail: sharada.rai@manipal.edu
The objectives of the study were to study the megakaryocytic morphology in JAK2 V617F positive MPN in different age groups and gender. The study focused on clinical manifestation, hematological parameters, megakaryocytic number, morphological types, pattern, localization, along with other hematopoietic cells, in bone marrow aspirates (BMAs) and bone marrow biopsies (BMBs). The correlation of the same was done with the final diagnostic entities.

\section{Subjects and Methods}

This was a retrospective and prospective hospital-based study, from January 2011 to May 2015 including a review of clinical features, hematological parameters, peripheral smear, BMA, and BMB in those cases of MPNs which were JAK2 V617F mutation positive. The study was conducted in Department of Pathology, Kasturba Medical College, Mangalore, Karnataka, India. A total of 15 cases of JAK2 V617F positive MPN were collected during this period. Clearance from Institutional Ethics Committee was taken.

The cases on which JAK2 V617F mutation analysis was performed were included in the study. The cases without JAK2 V617F mutation analysis were excluded from the study.

The peripheral smear and BMA smears were stained by Leishman stain, and BMBs were stained by routine hematoxylin and eosin ( $\mathrm{H}$ and $\mathrm{E}$ ), and Gomori's method was used for reticulin pattern. Reticulin stain was graded as per WHO 2008 guidelines. ${ }^{[1]}$

The peripheral smear, BMA smears, and BMB slides were studied with special emphasis on megakaryocytic morphology and pattern. Cytogenetic test JAK2 617F qualitative mutation analysis was done by polymerase chain reaction and gel electrophoresis. Specific megakaryocytic morphology definitions were considered for the study. ${ }^{[5]}$

This is an open access article distributed under the terms of the Creative Commons Attribution-NonCommercial-ShareAlike 3.0 License, which allows others to remix, tweak, and build upon the work non-commercially, as long as the author is credited and the new creations are licensed under the identical terms.

For reprints contact: reprints@medknow.com

How to cite this article: Ghai S, Rai S. Megakaryocytic morphology in Janus kinase 2 V617F positive myeloproliferative neoplasm. South Asian J Cancer $2017 ; 6: 75-8$. 
The data were analyzed by proportions, tables, and graphs. Various morphological features were analyzed for their frequency and were compared with the final diagnosis using Chi-square value and Fisher test, wherever appropriate. $P<0.05$ was considered to be significant. SPSS version 13.0 (IBM SPSS statistics) was used for the analysis.

\section{Results}

The study duration of $4 \frac{1}{2}$ years documented a total of 15 cases diagnosed as BCR-ABL negative JAK2 V617F positive MPN on complete blood count, peripheral smear examination, BMA, and BMB. Seven out of the 15 cases studied, seven cases $(46.6 \%)$ were of PV, three cases $(20 \%)$ of ET, and five cases $(33.3 \%)$ were of MF.

The age group affected in PV (71.4\%), ET (66.7\%), and MF (60\%) was between 40 and 60 years. One case $(20 \%)$ of MF belonged to age group $>60$ years. Male gender was affected predominantly in all the three disease entities with male:female ratio of $6: 1$ in PV, 2:1 in ET, and 3:2 in MF.

The most common symptom found was weakness in six out of seven cases of PV (85.7\%), one case of ET (33.3\%), and all five cases of MF (100\%). The patients of PV also presented with plethora in six out of seven cases $(85.7 \%)$. Other overlapping physical symptoms found were fatigue, abdominal pain, fever, weight loss, palpitation, giddiness, loss of appetite, and loose stools. The most common physical sign found was splenomegaly seen in three cases $(42.9 \%)$ of PV, two cases $(66.7 \%)$ of ET, and all the five cases $(100 \%)$ of MF.

The hematological parameters included in the study were hemoglobin, total red blood cell (RBC) count, total white blood cell (WBC) count, and platelet count. Mean value and range of hemoglobin, total RBC count, total WBC count, and platelet count are shown in Table 1.

Basophils were noted in all 15 cases $(100 \%)$ of JAK2 V617F positive MPNs. Six out of seven cases (85.7\%) of PV, all three cases of ET and one out of five cases (20\%) of MF showed giant platelets in peripheral smear. The circulating megakaryocytes were noted in one out of seven cases $(14.2 \%)$ of PV and one out of five cases (20\%) of MF.

All BMA and BMBs studied were hypercellular. Erythropoiesis was increased in all the seven cases of PV, one case of ET, and one case of MF. It was normal in remaining two cases $(66.7 \%)$ of ET and four cases (80\%) of MF. Myelopoiesis was increased in all the cases in both BMA and BMBs.

The most common finding of megakaryocytic morphology in PV, ET, and MF are shown in Table 2.

PV showed normal sized megakaryocytes, with cloud-like, hypolobated nuclei, small cytoplasm arranged in dense clusters. These features were similar to those seen in MF, with difference in reticulin fibrosis which was of Grade 2 in four cases of MF and Grade 3 in remaining case as compared to PV where reticulin grade of one was found predominantly. Features such as large size megakaryocytes with staghorn hyperlobated nuclei, abundant large cytoplasm, arranged in dense clusters were seen in ET. Reticulin grade was one in all the three cases of ET.

Comparison of megakaryocyte features as seen in different entities of JAK2 positive MPN in BMBs are shown in Table 3.

In BMA, PV, and MF along with normal sized megakaryocytes also showed the presence of few large sized megakaryocytes. In nuclear features, PV revealed the presence of cloud-like nuclei in four out of seven cases $(57.1 \%)$ and bare nuclei in three cases $(42.9 \%)$. Five out of seven cases $(71.4 \%)$ of PV showed hypolobated nuclei. In PV, three cases exhibited loose clustering, and another three showed dense clustering. Five out of seven cases (71.4\%) of PV showed the presence of small cytoplasm; one case showed (14.3\%) normal cytoplasm while one case (14.3\%) showed large cytoplasm. Few megakaryocytes with large cytoplasm were also noted in MF. These megakaryocytes are distributed in paratrabecular or perisinusoidal location.

\section{Discussion}

PV, ET, and MF are defined by WHO as distinct entities based on a combination of bone marrow morphology, genotype, clinical data, and phenotype. These are dynamic illnesses and can evolve into each other over time or progress toward MF. ${ }^{[4]}$ Our study of 15 JAK2 V617F positive MPNs constituted seven cases of PV, three cases of ET, and five cases of MF. The main highlight of these disorders is the presence of atypical, pleomorphic megakaryocytes. Hence, the emphasis of our study was to compare and correlate the morphological features of megakaryocytes in them.

A multidisciplinary approach is required while making a diagnosis of MPN. The modality of treatment, as well as prognosis, differs in all the entities and hence it is mandatory to make a correct diagnosis. ${ }^{[6]}$ Thrombocythemia prefibrotic phase of MF and other disorders with thrombocythemia (PV and ET) pose a diagnostic challenge and must be discriminated as these disorders have varied progression and complications. ${ }^{[6]}$

In our study, we analyzed the complete blood count along with BMA and BMBs with a special emphasis on megakaryocytic features.

The mean value of hemoglobin was found to be $18.9 \mathrm{mg} / \mathrm{dl}$ in PV, which was highest among the three entities. The mean platelet count $\left(854 \times 10^{9} / \mathrm{L}\right)$ was found to be the highest in ET. Patients in the pre-fibrotic phase of MF also showed high mean value of platelet resulting in problems of differentiating ET from MF as noted in other studies. ${ }^{[7-9]}$ The peripheral smear examination showed the presence of tear drop cells and nucleated RBCs in MF, crowding of RBCs in PV and platelet lakes in ET. Hence, complete blood count and peripheral smear examination could furnish the required clues for diagnostic modalities to be followed.

Table 1: Mean and range of hemoglobin, total red blood cell count, total white blood cell count, and platelet count

\begin{tabular}{|c|c|c|c|c|}
\hline \multirow[t]{2}{*}{ Diagnosis } & \multicolumn{4}{|c|}{ Mean (range) } \\
\hline & Hemoglobin (g/dl) & Total $\mathrm{RBC}$ count $\left(\times 10^{12} / \mathrm{L}\right)$ & Total WBC count $\left(\times 10^{9} / \mathrm{L}\right)$ & Platelet count $\left(\times 10^{9} / \mathrm{L}\right)$ \\
\hline PV & $18.9(18.4-19.8)$ & $6.8(5.25-8.12)$ & $14.81(8-21)$ & $426(214-806)$ \\
\hline ET & $12.9(11.1-15.6)$ & $5.4(4.56-6.64)$ & $20.67(11-28)$ & $854(608-1238)$ \\
\hline MF & $11.8(11-13.1)$ & $5.07(3.86-6.94)$ & $32.94(6-55)$ & $519(30-862)$ \\
\hline
\end{tabular}

$\mathrm{PV}=$ Polycythemia vera, $\mathrm{ET}=$ Essential thrombocythemia, $\mathrm{MF}=$ Myelofibrosis, $\mathrm{RBC}=$ Red blood cell, $\mathrm{WBC}=$ White blood cell 
Table 2: Features of megakaryocyte in subcategories of myeloproliferative neoplasm

\begin{tabular}{llllll}
\hline Disease & Size & Nuclei & Lobation & Clustering & Cytoplasm \\
\hline PV & Normal & Cloud-like & Hypolobation & Dense & Small \\
ET & Large & Staghorn & Hyperlobation & Dense & Large \\
MF & Normal & Cloud-like & Hypolobation & Dense & Small \\
\hline
\end{tabular}

$\mathrm{PV}=$ Polycythemia vera, ET=Essential thrombocythemia, MF=Myelofibrosis

Table 3: Comparison of megakaryocytic morphology in bone marrow biopsies in polycythemia vera, essential thrombocythemia and myelofibrosis bone marrow biopsies

\begin{tabular}{lccc} 
Features & PV (\%) & ET (\%) & MF (\%) \\
\hline Number & & & \\
Normal & 0 & 0 & 20 \\
Increased & 100 & 100 & 80 \\
Size & & & \\
Normal & 100 & 0 & 100 \\
Large & 0 & 100 & 0 \\
Nuclei & & & \\
Staghorn & 4.2 & 66.7 & 20 \\
Cloud-like & 79.2 & 33.7 & 60 \\
Dysmorphic & 8.3 & 0 & 0 \\
Bare nuclei & 8.3 & 0 & 20 \\
Hyperlobation & 14.3 & 100 & 20 \\
Hypolobation & 85.7 & 0 & 80 \\
Clustering & & & \\
Loose & 42.9 & 33.7 & 40 \\
Dense & 57.1 & 66.7 & 60 \\
Normal & 0 & 33.3 & 0 \\
Cytoplasm & & & \\
Small & 85.7 & 0 & 0 \\
Large & 14.3 & 66.7 & 0 \\
0 & 14.3 & 0 &
\end{tabular}

$\mathrm{PV}=$ Polycythemia vera, ET=Essential thrombocythemia, $\mathrm{MF}=$ Myelofibrosis

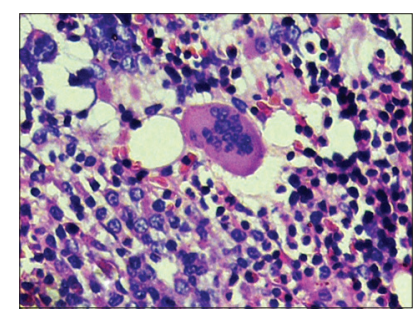

Figure 1: Megakaryocyte with hyperlobated staghorn nuclei

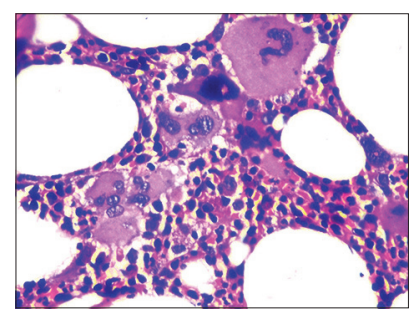

Figure 2: Megakaryocytes showing dense clustering with cloud-like nuclei in myelofibrosis
BMA and BMBs were marked by trilineage hypercellularity in all categories of MPN except one case of MF, which exhibited marked reticulin fibrosis. The presence of markedly hypercellular marrow as similar to other studies. ${ }^{[10-14]}$

The megakaryocytes in PV were normal sized admixed with few large and small megakaryocytes dispersed in both dense (three out of seven cases, 42.9\%) and loose clusters (three out of seven cases, $42.9 \%$ ) in PV with cloud-like hypolobated nucleus. Whereas megakaryocytes in ET exhibited large size, abundant mature cytoplasm with staghorn, hyperlobated nuclei. The presence of a characteristic nuclear feature, including predominance of staghorn nuclei in ET and cloud-like nuclei in PV, can help us to differentiate between the two entities. Other features may be overlapping. Few overlapping features included the presence of normal sized megakaryocytes in both ET and PV as seen by study of Gianelli et al. ${ }^{[15]}$ and Vytrva et al. ${ }^{[16]}$

Although ET demonstrates characteristic megakaryocyte morphology, patient of ET with a history of hemorrhage can show significant erythroid hyperplasia along with granulocytic proliferation and can be confused with early stages of PV. However, characteristic large megakaryocyte with staghorn, hyperlobated nuclei would favor a diagnosis of ET [Figure 1]. ${ }^{[1,17,18]}$

Megakaryocytes in prefibrotic phase of MF [Figure 2] were of normal size and were predominantly dispersed in dense clusters (three out of five cases, 60\%) and exhibited small cytoplasm with cloud-like (three out of five cases, $60 \%$ ) nuclei. These megakaryocytes are distributed in paratrabecular or perisinusoidal location. Sinusoidal dilatation was noted along with extramedullary hematopoiesis suggestive of MF with myeloid metaplasia. Similar findings were reported by other authors. ${ }^{[19,20]}$ Coexistence of normal sized megakaryocytes and cloud-like, hypolobated nuclei with distribution in dense clusters in the cellular phase of MF and PV can lead to difficulty in differentiating the two entities as reported by Vytrva et al. ${ }^{[16]}$ However, in the presence of leukoerythroblastic blood picture, dense clustering of normal sized megakaryocytes with cloud-like nuclei and few staghorn nuclei is more likely to point toward the diagnosis of MF as seen in our study. These findings would also be useful in patients with thrombocytosis in the early stage of MF where the differentiation from ET may be difficult.

The most probable reason for the morphological overlap is the JAK2 V617F mutation. There is a relationship between disease manifestations and allele burden of JAK2 V617F; the higher the copy number of mutated JAK2 V617F, the more likely disease resembles PV. Homozygosity for mutated JAK2, which results from mitotic recombination, is commonly found in PV, often in MF, and almost never in ET. Additional genetic abnormalities cooperate to initiate and influence the disease process. These reasons can be applied for the varying phenotypic aspects of these disorders. ${ }^{[21]}$

\section{Conclusions}

Megakaryocytes exhibited specific morphological features in the various categories of MPNs. PV was characterized by normal sized megakaryocytes, arranged in dense clusters, with small cytoplasm and hypolobated cloud-like nuclei. In contrast, ET exhibited giant megakaryocytes, abundant mature large cytoplasm, staghorn, hyperlobated nuclei in dense clusters. The megakaryocytes in MF were arranged in dense clusters, paratrabecular, or perisinusoidal in location, with small cytoplasm and hypolobated nuclei. As JAK2 V617F mutation is seen only in a subset of MPN understanding of specific megakaryocytic morphologies in various categories of MPN could enable accurate subclassification of MPN.

\section{Limitation of the study and future prospective}

This study needs to be validated in a larger group of people. Patients who are negative for JAK2 $617 \mathrm{~F}$ mutation but positive for JAK2 exon 12 mutation need to be studied further. The relative importance of megakaryocytic morphology can be made useful in cases of ET with specific features which differ from the comparable features seen in PV and MF.

\section{Financial support and sponsorship}

Nil. 


\section{Conflicts of interest}

There are no conflicts of interest.

\section{References}

1. Swerdlow SH, Campo E, Harris NL, Jaffe ES, Pileri SA, Stein H, et al. WHO Classification of Tumours of Haematopoietic and Lymphoid Tissue. Lyon: International Agency for Research on Cancer; 2008.

2. Campbell PJ, Green AR. The myeloproliferative disorders. N Engl J Med 2006;355:2452-66.

3. Vardiman JW, Thiele J, Arber DA, Brunning RD, Borowitz MJ, Porwit A, et al. The 2008 revision of the World Health Organization (WHO) classification of myeloid neoplasms and acute leukemia: Rationale and important changes. Blood 2009;114:937-51.

4. Anastasi J, Vardiman JW. Chronic myelogenous leukemia and the chronic myeloproliferative diseases. In: Knowles DM, editor. Neoplastic Hematopathology. $3^{\text {rd }}$ ed. Philadelphia: Lippincott Williams and Wilkins; 2014. p. 1108-36.

5. Koopmans SM, Bot FJ, Lam KH, van Marion AM, de Raeve H, Hebeda KM. Reproducibility of histologic classification in nonfibrotic myeloproliferative neoplasia. Am J Clin Pathol 2011;136:618-24.

6. Tripodo C, Valenti C, Ballarò B, Rudzki Z, Tegolo D, Di Gesù V, et al. Megakaryocytic features useful for the diagnosis of myeloproliferative disorders can be obtained by a novel unsupervised software analysis. Histol Histopathol 2006;21:813-21.

7. Passamonti F, Malabarba L, Orlandi E, Baratè C, Canevari A, Brusamolino E, et al. Polycythemia vera in young patients: A study on the long-term risk of thrombosis, myelofibrosis and leukemia. Haematologica 2003;88: 13-8.

8. Mossuz P, Girodon F, Donnard M, Latger-Cannard V, Dobo I, Boiret N, et al. Diagnostic value of serum erythropoietin level in patients with absolute erythrocytosis. Haematologica 2004;89:1194-8.

9. Marchioli R, Finazzi G, Landolfi R, Kutti J, Gisslinger H, Patrono C, et al. Vascular and neoplastic risk in a large cohort of patients with polycythemia vera. J Clin Oncol 2005;23:2224-32.

10. Thiele JM, Kvasnicka HM. Diagnosis of polycythemia vera based on bone marrow pathology. Curr Hematol Rep 2005;4:218-23.

11. Hattori N, Fukuchi K, Nakashima H, Maeda T, Adachi D, Saito B, et al. Megakaryopoiesis and platelet function in polycythemia vera and essential thrombocythemia patients with JAK2 V617F mutation. Int J Hematol 2008;88:181-8.
12. Michiels JJ, Kutti J, Stark P, Bazzan M, Gugliotta L, Marchioli R, et al. Diagnosis, pathogenesis and treatment of the myeloproliferative disorders essential thrombocythemia, polycythemia vera and essential megakaryocytic granulocytic metaplasia and myelofibrosis. Neth J Med 1999;54:46-62.

13. Michiels JJ, Thiele J. Clinical and pathological criteria for the diagnosis of essential thrombocythemia, polycythemia vera, and idiopathic myelofibrosis (agnogenic myeloid metaplasia). Int J Hematol 2002;76: 133-45.

14. Mesa RA, Silverstein MN, Jacobsen SJ, Wollan PC, Tefferi A. Population-based incidence and survival figures in essential thrombocythemia and agnogenic myeloid metaplasia: An Olmsted county study, 1976-1995. Am J Hematol 1999;61:10-5.

15. Gianelli U, lurlo A, Vener C, Moro A, Fermo E, Bianchi P, et al. The significance of bone marrow biopsy and JAK2V617F mutation in the differential diagnosis between the "early" prepolycythemic phase of polycythemia vera and essential thrombocythemia. Am J Clin Pathol 2008; 130:336-42.

16. Vytrva N, Stacher E, Regitnig P, Zinke-Cerwenka W, Hojas S, Hubmann E, et al. Megakaryocytic morphology and clinical parameters in essential thrombocythemia, polycythemia vera, and primary myelofibrosis with and without JAK2 V617F. Arch Pathol Lab Med 2014; 138:1203-9.

17. Kvasnicka HM, Thiele J. Bone marrow angiogenesis: Methods of quantification and changes evolving in chronic myeloproliferative disorders. Histol Histopathol 2004; 19: 1245-60.

18. Lundberg LG, Lerner R, Sundelin P, Rogers R, Folkman J, Palmblad J. Bone marrow in polycythemia vera, chronic myelocytic leukemia, and myelofibrosis has an increased vascularity. Am J Pathol 2000; 157: 15-9.

19. Mesa RA, Hanson CA, Rajkumar SV, Schroeder G, Tefferi A. Evaluation and clinical correlations of bone marrow angiogenesis in myelofibrosis with myeloid metaplasia. Blood 2000;96:3374-80.

20. Thiele J, Kvasnicka HM. Hematopathologic findings in chronic idiopathic myelofibrosis. Semin Oncol 2005;32:380-94.

21. Brousseau M, Parot-Schinkel E, Moles MP, Boyer F, Hunault M, Rousselet MC. Practical application and clinical impact of the WHO histopathological criteria on bone marrow biopsy for the diagnosis of essential thrombocythemia versus prefibrotic primary myelofibrosis. Histopathology 2010;56:758-67. 